\title{
INFÂNCIAS, CULTURA VISUAL E CONSUMO: REFLEXÕES DE PESQUISAS
}

\author{
CHILDHOOD, VISUAL CULTURE AND CONSUMPTION: \\ STUDIES THOUGHTS
}

Adriana Hoffmann ${ }^{1}$ (D)

RESUMO: O artigo reflete sobre pesquisas realizadas nos últimos vinte anos, com foco nas relações entre infância, cultura visual e consumo. As pesquisas aqui trazidas, na linha da cultura visual, investigaram não somente as imagens, mas principalmente as relações das crianças com e pelas imagens. Apontam como as crianças "são" com os artefatos da cultura visual. Com a conexão de nossas vidas com as imagens hoje vivemos "experiências culturais de olhar" e o desafio dessa reflexão é pensar o modo como as crianças lidam com essa "visualização da existência" em meio ao consumo vivido por elas.

Palavras-chave: Cultura visual. Formação. Infância.

\begin{abstract}
The article conveys some thoughts about the studies conducted along the last twenty years, focusing on the relationships between childhood, visual culture, and consumption. Those studies, embedded in the visual culture field, investigate not just images, but specially children's interactions with and through the images. It points how children 'are' with the artifacts of visual culture. For we are connected with many images today, we live 'cultural experiences of seeing, and the challenge to figure how our children handle this 'existential sightseeing' in the midst of the consumption lived by them.
\end{abstract}

Keywords: Visual culture. Development. Childhood.

\footnotetext{
1.Universidade Federal do Estado do Rio de Janeiro - UNIRIO - Rio de Janeiro (RJ), Brasil.

*Autora correspondente: profadrihoff@gmail.com

Número temático organizado por: Gilka Girardello, Adriana Hoffmann e Inês Vitorino Sampaio

- Esse artigo é fruto do projeto de pesquisa "A VISUALIDADE DA CULTURA E A EDUCAÇÃO: ESTUDOS SOBRE OS MODOS DE VIVER/PRODUZIR/APRENDER COM A IMAGEM NA CONTEMPORANEIDADE” financiado pela FAPERJ por meio do Edital Jovem Cientista do Nosso Estado de 2017 sob a coordenação da pesquisadora autora do referido artigo.
} 


\section{Introdução}

O presente texto narra um percurso reflexivo que resgata várias pesquisas realizadas acerca das relações das crianças com as imagens midiáticas. Tais pesquisas, aos poucos, deram origem ao grupo de pesquisa CACE (Comunicação, Audiovisual, Cultura e Educação), da Universidade Federal do Estado do Rio de Janeiro (Unirio) ${ }^{1}$. O grupo - coordenado por mim - dedica-se a continuar esse dialogo iniciado nos meus estudos de mestrado, doutorado e pós-doutorado, a partir dos quais propus, até o momento, três projetos institucionais.. Trago, neste artigo, as reflexões realizadas a partir das pesquisas anteriores à criação do grupo e, também, alguns estudos surgidos dentro do grupo, puxando fios de diálogo das pesquisas implementadas com as infâncias nesse contexto coletivo de produção.

Uma questão que tem me intrigado nas pesquisas que venho fazendo nos últimos vinte anos diz respeito à relação cada vez maior das crianças com as narrativas pela imagem, em diferentes mídias (livros, TV, cinema, Internet, dispositivos móveis, entre outros). A preponderância do ver, da imagem e de sua difusão nos modos de narrar e consumir narrativas visuais torna-se parte integrante de nossa cultura cotidiana.

Ao mesmo tempo que o consumo de uma imensa quantidade de imagens já se tornou prática regular, e muitas pessoas já acordam olhando para imagens nas pequenas telas dos seus celulares, vemos também que as inundações de imagens que nos chegam diariamente acabam por nos colocar em situações antes vividas tendo aparelhos de televisão ligados o dia inteiro. No entanto, hoje, as TVs estão nas palmas de nossas mãos.

Sabemos que, desde os anos 1950, com o advento da TV, começamos a ter cada vez mais a presença da imagem em nossas vidas. No entanto, seria possível imaginar que tais imagens se tornariam, como ocorre hoje, praticamente uma imersão diária? Seria possível pensar que as próprias crianças estariam imersas nesse contexto de visualidade constante? Como pensar essa situação hoje, numa pandemia em que as interações passam a ser obrigatoriamente pela imagem nas telas, em virtude da necessidade de isolamento físico?

O autor Chris Jenks, já nos idos de 1995, discutia como, na sociedade contemporânea, a imagem tornou-se o centro das referências do ser humano, já que tudo passa pela imagem e é significado por meio dela. Já naquela época, o autor deu a isso o nome ocularcentrismo ${ }^{2}$ : ter o olho ou a imagem como centro. O autor apontava como essa nova forma de representar a realidade podia, de fato, alterar nossa percepção. Segundo ele, a cultura moderna elegeu a visão como o primeiro meio para a comunicação, e o mundo moderno tornou-se um fenômeno visual. O autor, mesmo escrevendo na década de 1990, quando muitas das plataformas constituidoras das visualidades atuais (como Instagram, YouTube e Snapchat entre outras) ainda não existissem, leva-nos a pensar acerca do início de uma mudança cultural que veio se ampliando, caracterizando a "virada pictórica ou visual" apontada por Wulf e Hall. A questão central dessa virada sobre o que é a imagem soma-se às questões sobre como as imagens são usadas, o que fazemos com elas e o que elas fazem conosco (WULF, 2013).

Essa virada visual, com o tempo, começa a ampliar o debate dos Estudos Culturais, constituindo os estudos da Cultura Visual. Hernandez (2013) considera a cultura visual não somente uma atitude ou metodologia viva, mas um ponto de encontro entre o que seria um olhar cultural (visualidade) e as práticas de subjetividades que a ele se vinculam. Esse ponto de encontro permite pesquisar as relações entre os artefatos da cultura visual, aquele que vê (e é visto) e os relatos visuais que, por sua vez, constroem o visualizador. O autor sustenta duas posições: uma em que o relevante nas pedagogias da cultura visual não são os objetos, mas as relações que mantemos com eles; e outra que explora a noção de produtores da cultura visual dos indivíduos, na medida em que não se trata somente de fazer com, mas também de ser com as representações e artefatos da cultura visual. 
Mirzoeff (2016) complementa esse "ser com" a cultura visual ao discutir as dimensões políticas e éticas dos Estudos Culturais e defender que é preciso descolonizar o conhecimento e as mídias, reconhecendo as formas pelas quais elas são colonizadas. Ele entende a cultura visual como "uma tática para estudar as funções de um mundo abordado por via de imagens de todo o tipo de visualizações”, no qual o termo visualidade não se presta a designar simplesmente o que é visível, mas representa uma prática discursiva e reforça o poder com autoridade. Foi por discussões como essas, trazidas pelos autores aqui citados, entre outros, que muitas pesquisas ancoradas nos Estudos Culturais passaram a focar nos Estudos da Cultura Visual.

\section{O que as Pesquisas com as Crianças nos Fizeram Perceber e Pensar?}

Nas pesquisas realizadas por mim e por meu grupo, institucionalmente (FERNANDES, 2003, 2009, 2010, 2012, 2013, 2017, 2019; DALETHESE, 2013; GATTO, 2013; CORDEIRO, 2013; BATISTA, 2014; CELESTINO, 2015; DALETHESE, 2017; FOSSALUZA, 2018), algumas questões se destacam e dialogam nesse processo cultural de relação da criança com as imagens.

Quando o principal meio de acesso às histórias na cultura eram os livros, os mais procurados pelas crianças em nossas pesquisas eram aqueles que tinham imagens. O mercado editorial - ao longo dos anos - especializou-se em livros cada vez mais artísticos e belos na questão estética, tendo esse entrelaçamento entre texto e imagem na condução da narrativa. Os ilustradores passaram a ser cada vez mais valorizados e realmente reconhecidos como autores dos livros, tanto quanto os escritores. Texto e imagem passaram a ser percebidos pelas crianças como um só. Um livro sem imagens tinha, para as crianças participantes de nossas pesquisas, menos valor.

Essa prática de ler com e por imagens foi sendo cada vez mais apropriada pelas crianças. Em pesquisa realizada há cerca de dez anos (FERNANDES, 2009), as crianças me apontavam seu vínculo com o visual ao ler e ao contar as histórias. Muitas vezes, o contar dava-se pela narração a partir das imagens, mesmo para aquelas crianças já leitoras - no contar pelo ver; para outras, a escolha do que ler realizava-se pela imagem do livro, em afirmações como: "Gostei pela capa". Outras crianças ainda falavam de livros que não haviam lido, mas que diziam "conhecer só de ver". Essas referências apontam uma relação bem cotidiana das crianças com a visualidade presente nos livros. Esse costume de ver frequentemente as habilita a falar, com segurança, das histórias pelas imagens vistas.

Ao falarem que histórias sem imagens não têm graça, as crianças das pesquisas chamam a atenção para a necessidade de "contar com imagens", mostrando a forma como reconheciam as histórias pelas imagens, sem a necessidade de ler o texto escrito para falar a respeito delas. Ao mesmo tempo, as crianças faziam a associação entre livro e filme como "parecidos", ao dizerem que as histórias de que mais lembravam - de todas as que conheciam - eram aquelas presentes em livros e em filmes. Aos poucos, essa associação do contar com/por imagens, trazida pelas crianças nas pesquisas, fica cada vez mais evidente nas práticas vividas por elas em nossa sociedade. Hoje, elas contam com/por imagens no Instagram, no Snapchat, no YouTube e nos muitos espaços on-line nos quais a imagem se tornou a principal forma de trocar e comunicar-se uns com os outros.

Assim, há cerca de dez anos, as crianças já nos mostravam, com suas práticas, o quanto esse contar pela imagem, antes pensado como vinculado apenas ao livro, torna-se cada vez mais imagético e presente na sociedade atual, por meio de aplicativos e demandas da cultura capitalista. O que percebemos nessas práticas de consumo das crianças com as imagens é essa noção de que, ao consumirem e contarem pelas imagens, elas apontam seu papel de produtoras da cultura visual, pois não somente contam histórias com as imagens, mas também tornam-se sujeitos pelas relações (HERNANDEZ, 2013) que constroem com elas. 
Esse "ser com as imagens", como nos aponta Hernandez anteriormente, aparece na dimensão do vínculo das crianças com as imagens, como no caso de uma criança da pesquisa que disse "pensar em quadrinhos". Ao dizer que organizava seu pensamento e suas narrativas por imagens porque pensava em quadrinhos, essa criança nos mostra que o consumo cotidiano dela às leituras dos quadrinhos era mediador do seu processo de pensar e de expressar-se por imagens. Ela era exímia leitora e fã de vários quadrinhos, bem como tinha os quadrinhos como referência para suas histórias. Esse modo de pensar, mediado pela imagem mais do que pelo texto, já era constitutivo dos "modos de ser" e ver dela e de outras que - como ela - fossem leitoras ávidas de histórias em quadrinhos.

Em pesquisa anterior, no início dos anos 2000 (FERNANDES, 2003, 2012), outra criança aponta que, nas diferentes mídias por ela consumidas, "há trocas alternadas"; ou seja, diz, ao seu modo, que as mídias conversam entre si, que algo que ela viu na TV aparece na revista e vai migrando para outras mídias e viceversa, sendo algo que ela vai seguindo no percurso entre várias mídias, “de modo alternado”. No tempo dessa pesquisa - idos de 2003 -, esse menino parecia falar do que hoje chamamos de narrativa transmídia, tipo de narrativa que circula por diferentes suportes de mídia, complementando-se. Vai do programa ou seriado de TV para a revista, para os aplicativos e, depois, para o álbum de figurinhas ou o jogo, e assim por diante. Para saber mais acerca de uma determinada história, é necessário seguir essas trocas nas redes, por meio dessa narrativa transmidiática que agora foi apropriada pelo mercado. Nesse contexto, aquela imagem do livro hoje circula por aplicativos, sites e vídeos da Internet, transmidiaticamente, e as crianças perceberam isso rapidamente. Assim, o que as crianças apontam é algo que não se restringe somente à infância, mas a mudanças nos modos de relacionar-se com as mídias de toda a sociedade. São, como aponta Hernandez (2013), as relações entre aquele que vê (e é visto) e os artefatos da cultura visual que apontam como nos construímos como visualizadores dessa cultura.

Em falas atuais de crianças em pesquisas mais recentes, ao dizerem frases como "Só vejo a TV pela Internet"; "Só vejo TV pelo YouTube"; "Prefiro ver YouTube do que assistir TV"; "Não vejo mais TV, só Netflix ou YouTube", elas estão apontando as formas pelas quais se constituem como visualizadoras da cultura a partir dos seus modos de consumo. Uma das pesquisas que discutiram o consumo que as crianças fazem do YouTube foi a de Dalethese (2017). Tivemos um período em que os vídeos em DVD e CD eram intensamente consumidos pelas crianças nas TVs de suas casas. Atualmente, esses hábitos foram praticamente deslocados para o consumo de vídeos no YouTube, em dispositivos móveis como celulares, tablets, entre outros - principalmente por acessos sob demanda on-line.

Em outra pesquisa (GATTO, 2013) sobre o consumo que as crianças fazem dos filmes, percebeu-se que o acesso de algumas crianças a eles se dava, predominantemente, pelos materiais piratas e pela Internet, sendo raras as idas desse público ao cinema. Esse acesso das crianças aos filmes pela Internet, na pesquisa de Gatto, amplia-se cada vez mais. Vemos que, em 2020, cada vez mais as locadoras e locais de venda de CD e DVD deixam de existir, até mesmo nos lugares mais pobres; fecham suas portas porque não há consumidores suficientes que aluguem filmes para assistir em casa - muitas vezes nem mesmo nas LAN houses, espaços antes destinados ao público que não tinha acesso em casa.

A Netflix, o YouTube e outras plataformas passam a ser os locais de acesso on-line para o consumo de filmes - não somente para crianças, mas para toda a sociedade. As crianças, como produtoras de cultura, modificam seus modos de consumo. Nesse contexto, as pesquisas realizadas apontam que a escola, quando faz projetos com o cinema, torna-se mediadora do ver para os alunos; percebe-se, ao longo dos últimos dez anos, o aumento do número de programas e políticas de audiovisual que chegam à escola. Pesquisas como as de Fernandes (2010, 2013), Dalethese (2013), Cordeiro (2013), Gatto (2013), Celestino (2015), em nosso grupo, mostraram esse papel do assistir cinema e do produzir filmes nas escolas como espaço de formação 
para crianças e jovens que delas participam. Esse fazer cinema, oferecido nos espaços escolares, é bastante atrativo para as crianças, mas o maior vínculo dos que participaram de nossas pesquisas - em seu consumo privado - ainda continua sendo os vídeos da Internet.

Ao mesmo tempo, as pesquisas feitas apontam que esse consumo gera o desejo de também ser produtor de mídia. Como Mirzoeff destaca, a visualidade pelas mídias, muitas vezes, produz um processo de colonização, já que a visualidade não trabalha apenas com o que é visível, mas também denota poder, ao dar visibilidade a algumas coisas e invisibilizar outras. Percebe-se - a partir do que nos dizem as crianças nas pesquisas - uma migração dos sonhos. O antigo sonho das crianças de ser escritoras e escrever um livro já passou por quererem ser blogueiras e, atualmente, ter um canal e ser YouTubers - e até mesmo Instagramers. Nossas pesquisas foram acompanhando essas mudanças. Ao mesmo tempo, é nesse mesmo contexto que temos aplicativos e redes sociais (Pinterest, YouTube, Instagram, Snapchat, entre outros mais recentes) que têm a visualidade como mote principal de troca e narrativa entre os sujeitos. Amplia-se "o contar pelo ver", de que as crianças falam nas pesquisas anteriores.

Esse "contar pelo ver" passa também pela performance de quem conta - como aponta por Paula Sibilia $(2008$, 2012) -, por exemplo nos canais do YouTube, que têm objetivo diferente do cinema. A performance é cada vez mais perceptível no universo e nas práticas de crianças, passando até mesmo a ser incorporada por empresas que, para vender seus produtos, convidam crianças a fazer vídeos, por exemplo, em que brincam com brinquedos para vendê-los.

Ao mesmo tempo, boa parte daquilo em que as crianças veem e produzem nesses novos espaços de visualização é também composta de vídeos em que o "contar pelas imagens" relaciona-se ao performar e exibir-se pelo brincar ou pelo consumir algo valorizado (vídeos de maquiagem, roupas e situações do cotidiano, entre outros). Nesse contexto, algumas crianças brincam filmando-se. Isso apareceu nas pesquisas de Dalethese (2017) e Fossaluza (2018). O falar para a câmera e pensar no público já se torna um dos elementos da brincadeira das crianças - brincadeira com foco em quem verá seu vídeo, já com aspecto de show do eu $u^{4}$, como discutido por Sibilia. O performar, comentado por Sibilia, já passa a ser prática cotidiana, no tirar fotos e filmar diferentes momentos de sua vida, o que pensam e o que fazem as crianças. Algumas crianças vivem performaticamente suas brincadeiras através das visualidades dos vídeos.

Susana Cunha observa que os Estudos da Cultura Visual refletem e analisam "como o universo visual (aquilo que se vê) e a visualização (os modos de ver e as tecnologias da visão) estão nos constituindo" (CUNHA, 2005), indo muito além de simplesmente se ocupar dos artefatos visuais, pois se preocupam "em como as imagens são produzidas, distribuídas e utilizadas socialmente, como uma prática cultural que produz e negocia significados" (CUNHA, 2005, p. 43). A autora cita Hernández, para quem a importância primordial da cultura visual é mediar o processo de como olhamos e como nos olhamos, e contribuir para a produção de mundos (HERNÁNDEZ, 2000, p. 52 apud CUNHA, 2005, p. 43).

Temos visualidades construídas na relação com produção, consumo e partilha de bens visuais na cultura. Entendemos que vivemos em um contexto no qual há cada vez mais uma ênfase na centralidade do visual nas formações culturais, numa condição em que a cultura está imersa nesse reino da imagem (CAMPOS, 2012). Nesse sentido, a cultura visual é, segundo esse autor, tanto um "repositório visual", relacionado a contextos coletivos, quanto um modo de produzir, apreender e decodificar visualmente a realidade - um campo de visibilidade. Sabemos que a cultura visual hoje não existe sem se articular às tecnologias e aos dispositivos midiáticos. Desse modo, falar de imagem hoje é falar de "imagem midiática" (JOLY apud CAMPOS, 2012). Portanto, ao resgatarmos as pesquisas anteriores, vemos como as crianças, imersas nesse contexto da cultural visual, relacionam-se, produzem e se apropriam da cultura pela imagem midiática, construindo seus mundos. A visualidade contemporânea compreende todo um campo de visibilidade e 
produções de sentido acerca das imagens que circulam e que definem, de acordo com Campos (2013), quem pode ver; com que tecnologias e de que modo se constroem conhecimentos visuais sobre o que se vê. Mirzoeff (2016) nos ajuda a pensar, a partir das pesquisas, em como se dá esse processo de "visualização da existência" pelas práticas de consumo das crianças.

Como essa visualidade da cultura, cada vez mais ampla, exprimindo-se nos consumos e nas formas de narrar cotidianas das crianças - ao sonharem ser YouTubers e brincarem filmando-se - repercute na escola? As visualidades fazem parte do modo como a sociedade organiza-se. Tornam-se algo estrutural. Os movimentos sociais, as aprendizagens e os registros da história estão sendo construídos a partir das imagens. Como essas lógicas e formas de pensar visualmente podem articular-se na formação das crianças? Que novos diálogos buscaremos construir nesse contexto?

\section{Considerações Finais}

Entendo que esse contexto visual que nos provoca pode trazer possibilidades interessantes se soubermos dialogar com ele, percebendo as oportunidaes de criação de conhecimentos, de cultura, de mobilização e de problematização. Sempre há o que discutir, o que problematizar e o que refletir para fazer escolhas e se posicionar. Todavia, reconhecer esse contexto e dialogar com ele requer experimentá-lo, não aboli-lo.

Os estudos do Comitê Gestor da Internet, nas pesquisas TIC Kids Online, em sua última edição, publicada on-line em 2019, apontam que uma das atividades preferidas pelas crianças na Internet é o consumo de vídeos (77\% de seu uso). Segundo relatório dessa consultoria, estima-se que, naquele ano, os vídeos já eram mais populares do que as redes sociais, apontando que, em alguns anos, teremos $80 \%$ do público assistindo a vídeos como principal atividade ao acessar a Internet. Ver vídeos cotidianamente pela Internet já se tornou "um sintoma" da cultura, no sentido que discutem Rosa Fischer (2008) e Maria Rita Kehl (2005). A mediação do mundo torna-se mais visual do que nunca.

Há, em todo o Brasil, por exemplo, cerca de doze leis (estaduais e municipais), como apontam Rodrigues, Segundo e Ribeiro (2018), que proíbem o uso de celular e de redes móveis na escola. No contexto de uma pandemia como a que estamos vivendo com o coronavírus, em que o isolamento tornou-se uma demanda e as crianças deixaram de ir presencialmente à escola, o celular tornou-se, muitas vezes, o único meio de contato com outros e, até mesmo, de contato com a própria escola. O novo contexto visibilizou a presença da imagem como estruturante dessa sociedade capitalista. A conexão com o outro pela imagem, seja por WhatsApp, seja por aplicativos de videoconferência, seja por outras plataformas, só reforça o fato de que a visualidade já se concretizou no cotidiano da sociedade e das práticas das crianças, como trouxemos neste artigo.

A pesquisa de Fossaluza (2018) apontou que as crianças, pelas suas práticas, relacionam o uso das imagens artesanais com as tecnológicas quando tem essa oportunidade e essa experiência. Tal pesquisa aponta, também, como esse entrelaçamento traz potencias para diálogos e criações. Nesse sentido, uma extensão possível a ser pensada é essa potencialização dos espaços de diálogo pelo visual como troca de experiências. A pandemia atual traz questões para ampliar esse debate.

Qual é o papel do outro na cultura atual além das curtidas e dos seguidores das redes? Como percebemos nossa relação com esse outro por meio da imagem - agora diariamente, na pandemia - nas plataformas de videoconferência utilizadas até mesmo pelas escolas? Como poderíamos refletir e potencializar o diálogo nesse contexto de contar com imagens/visualidades, como falam as crianças? Como essa presença da imagem afeta as formas de narrar na escola? Como as pesquisas anteriores e o contexto atual nos permitem pensar articulações entre memória, tempo e narrativa nesse contexto de visualidade compartilhada? Qual é 
a lógica do tempo narrativo no contexto atual? Como fazer esse diálogo entre o artesanal e o tecnológico no contexto da escola e da vida? Como a imagem on-line está constituindo nossas crianças?

São todas essas questões que nos desafiam e para as quais não temos respostas. Seguimos perguntando, refletindo e pesquisando sobre o que essas mudanças provocam em nosso pensar para também provocar o nosso fazer de modo mais potente em nossas práticas e em nossas pesquisas.

\section{Notas}

1. Site do grupo de pesquisa CACE, com as pesquisas realizadas: https://caceunirio.wixsite.com/cace

2. Destaque para o termo usado pelo autor Chris Jenks em seu livro de 1995.

3. Termo usado por mim nas pesquisas a partir da fala das crianças sobre o "contar pelo ver" na relação delas com os livros por meio das imagens presentes neles. Para saber mais, a pesquisa mais detalhada foi publicada no livro Narrativas das Crianças na Sociedade da Imagem, publicado pela pela editora Appris.

4. Termo cunhado por Paula Sibilia, publicado no livro homônimo.

5. O termo "sintoma da cultura" é discutido pela autora Maria Rita Kehl.

\section{REFERÊNCIAS}

BATISTA, L. F. S. Jovens YouTubers: processos de autoria e aprendizagens contemporâneas. 2014. Dissertação (Mestrado em Educaçao) -Programa de Pós-graduação em Educação, Universidade Federal do Estado do Rio de Janeiro, Rio de Janeiro, 2014.

CAMPOS, R. A cultura visual e o olhar antropológico. Revista Visualidades, Goiânia, v. 10, n. 1, jan.-jun. 2012. https://doi.org/10.5216/vis.v10i1.23083

CELESTINO, R. G. As crianças e suas narrativas audiovisuais: Descobertas no decorrer de uma pesquisa. 2015. Dissertação (Mestrado em Educação) - Programa de Pós-graduação em Educação, Universidade Federal do Estado do Rio de Janeiro, Rio de Janeiro, 2015.

COMITÊ GESTOR DA INTERNET NO BRASIL. Pesquisa sobre o uso de internet por crianças e adolescentes no Brasil: TIC Kids Online Brasil 201 acertei as datas que estavam erradas.. São Paulo: CETIC. br, 2018. Disponível em: https://cetic.br/media/docs/publicacoes/2/tic_kids_online_2018_livro_eletronico. pdf. Acesso em: 2019.

CORDEIRO, K. M. Cinema e juventude: relações criadas pelos jovens dentro e fora da escola. 2013. Dissertação (Mestrado em Educação) - Programa de Pós-graduação em Educação, Universidade Federal do Estado do Rio de Janeiro, Rio de Janeiro, 2013.

CUNHA, S. R. V. Educação e cultura visual: uma trama entre imagens e infância. 2005. Tese (Doutorado em Educação) - Faculdade de Educação, Universidade Federal do Rio Grande do Sul, Porto Alegre, 2005. 
DALETHESE, T. Cinema, narrativa e experiências: a formação atravessada pela prática cineclubista na Universidade. 2013. Monografia de conclusão de curso (Licenciatura em Pedagogia) - Universidade Federal do Estado do Rio de Janeiro, Rio de Janeiro, 2013.

DALETHESE, T. Faz de conta que todos nós somos YouTubers: crianças e narrativas contemporâneas. 2017. Dissertação (Mestrado em Educação) - Programa de Pós-graduação em Educação, Universidade Federal do Estado do Rio de Janeiro, Rio de Janeiro, 2017.

FERNANDES, A. H. As mediações na produção de sentido das crianças sobre os desenhos animados. 2003. 178 f. Dissertação (Mestrado em Educação) - Departamento de Educação, Pontifícia Universidade Católica do Rio de Janeiro. Rio de Janeiro, 2003.

FERNANDES, A. H. Infância e cultura: o que narram as crianças na contemporaneidade? 2009. 241 f. Tese (Doutorado em Educação) - Programa de Pós- graduação em Educação, Universidade do Estado do Rio de Janeiro, Rio de Janeiro, 2009.

FERNANDES, A. H. O cinema e as narrativas de crianças em diferentes contextos educativos. Projeto de Pesquisa. Rio de Janeiro: Unirio/FAPERJ, 2010.

FERNANDES, A. H. As crianças e os desenhos animados: mediações nas produções de sentidos. Rio de Janeiro: Editora Nau, 2012.

FERNANDES, A. H. O Cinema e as narrativas na era da convergência: modos de consumo, formação e produção de audiovisuais de crianças, jovens e professores. Projeto de Pesquisa. Rio de Janeiro: Unirio/ FAPERJ, 2013.

FERNANDES, A. H. A visualidade da cultura contemporânea e a educação: estudos sobre os modos de viver/produzir a imagem. Projeto de Pesquisa. Rio de Janeiro: Unirio/FAPERJ, 2017.

FERNANDES, A. H. As narrativas das crianças na sociedade da imagem. Curitiba: Appris, 2019.

FISCHER, R. Mídia, juventude e memória cultural. Educação \& Sociedade. Campinas, v. 29, n. 104, p. $667-$ 686, out. 2008. https://doi.org/10.1590/S0101-73302008000300003

FOSSALUZA, D. Narrativas das crianças com tapetes tridimensionais de histórias: Entre as dimensões artesanais e tecnológicas. 2018. Dissertação (Mestrado em Educação) - Programa de Pós-graduação em Educação, Universidade Federal do Estado do Rio de Janeiro, Rio de Janeiro, 2018.

GATTO, E. R. Narrativas das crianças com os filmes: reflexões sobre infância e consumo a partir do cineclube Megacine. 2013. Dissertação (Mestrado em Educação) - Programa de Pós-graduação em Educação, Universidade Federal do Estado do Rio de Janeiro, Rio de Janeiro, 2013.

HALL, S. A centralidade da cultura: notas sobre as revoluções culturais do nosso tempo. Educação \& Realidade, Porto Alegre, v. 22, n. 2, p. 15-46, jul.-dez. 1997. Disponível em: https://seer.ufrgs.br/educacaoerealidade/ article/view/71361/40514 Acesso em: 2019. 
HERNANDEZ, F. Pesquisas com imagens, pesquisar sobre imagens: revelar aquilo que permanece invisível nas pedagogias da cultura visual. In: MARTINS, R.; TOURINHO, I. Processos e práticas de pesquisa em Cultura visual e educação. Santa Maria: EdUFSM, 2013.

KEHL, M. R. Juventude como sintoma da cultura. In: NOAVES, R.; VANNUCHI, P. (orgs.). Juventude e sociedade: trabalho, educação, cultura e participação. São Paulo: Perseu Abramo, 2005. p. 89-113.

JENKS, C. The centrality of the eye in western culture. Visual culture. New York: Routledge, 1995.

MIRZOEFF, N. Como ver el mundo: uma nueva introducion a la cultura visual. Barcelona: Paidós, 2016.

RODRIGUES, F.; SEGUNDO, G.; RIBEIRO, L. M. O uso de celular na sala de aula e a legislação vigente no Brasil. In: CONGRESSO SOBRE TECNOLOGIAS NA EDUCAÇÃO, 3., 2018, Fortaleza. Anais [...]. Fortaleza: Cultura Maker na Escola, 2018. Disponível em: http://ceur-ws.org/Vol-2185/CtrlE_2018_paper_32.pdf Acesso em: jun. 2019.

SIBILIA, P. O show do Eu - a intimidade como espetáculo. Rio de Janeiro: Nova Fronteira, 2008.

SIBILIA, P. Redes ou paredes: a escola em tempos de dispersão. Rio de Janeiro: Contraponto, 2012.

WULF, C. Homo Pictor: imaginação, ritual e aprendizado mimético no mundo globalizado. São Paulo: Hedra, 2013. 